\title{
Modelamiento de los arrollamientos para determinación de las tensiones transitorias transferidas en un transformador de potencia
}

\section{Modeling of the windings for determining the transient voltages transferred in a power transformer}

\author{
Jorge Carmelo Ramos Carrión ${ }^{*}$, Eduardo David Arrollo Villanueva1* \\ ${ }^{1}$ Facultad de Ingeniería Eléctrica y Electrónica, Universidad Nacional de Ingeniería, Av. Túpac Amaru 210 Lima, Perú
}

Recibido (Recieved): 03/11/2018 Aceptado (Accepted): 06/06/2019

\begin{abstract}
RESUMEN
El presente artículo expone el desarrollo de la investigación sobre el modelamiento de los arrollamientos de un transformador de potencia para determinar y analizar las tensiones transitorias transferidas al arrollamiento secundario cuando se aplica una tensión tipo impulso atmosférico al arrollamiento primario. Se modela cada arrollamiento considerando su forma geométrica y constructiva por medio de parámetros eléctricos concentrados - Resistencia, Conductancia Dieléctrica, Capacitancias Serie, Capacitancia a Tierra, Inductancia Propia e Inductancia Mutua - calculados con formulaciones convencionales, los que se incorporan a un modelo integral de los arrollamientos que explica su comportamiento frente a sobretensiones. El análisis se realiza con el apoyo del software ATP Electromagnetic Transients Program, ATP Draw, lográndose demostrar la utilidad del modelo ya que se pueden calcular, al interior de los arrollamientos, las tensiones transitorias propagadas y transferidas, permitiendo el estudio de los esfuerzos dieléctricos en los aislamientos de los arrollamientos para mejorar su dosificación y geometría en el proceso de diseño del transformador de potencia.
\end{abstract}

Palabras clave: Modelamiento de arrollamientos, tensiones transitorias propagadas y tensiones transitorias transferidas.

\section{ABSTRACT}

The present article exposes the development of the research on the modeling of the windings of a power transformer to determine and analyze the transient voltages transferred to the secondary winding when an atmospheric impulse voltage is applied to the primary winding. Each winding is modeled considering its geometric and constructive form by means of concentrated electrical parameters - Resistance, Dielectric Conductance, Series Capacitances, Earth Capacitance, Inductiveness and Mutual Inductance - calculated with conventional formulations, which are incorporated into an integral model of the windings that explains his behavior in front of surges. The analysis is carried out with the support of ATP Electromagnetic Transients Program software, ATP Draw, being able to demonstrate the utility of the model since it is possible to calculate, inside the windings, the transient voltages propagated and transferred, allowing the study of the stresses dielectrics in the insulation of the windings to improve their dosage and geometry in the design process of the power transformer.

Keywords: Modeling of windings, propagated transient voltages and transient voltages transferred.

\footnotetext{
* Corresponding author:

E-mail: jorgeramosc@uni.edu.pe, earroyo@uni.pe 


\section{INTRODUCCIÓN}

Las sobretensiones en los sistemas eléctricos de potencia generalmente han sido estudiadas desde el punto de vista del diseño del aislamiento o del equipo de protección. En los últimos años, la tendencia a utilizar tensiones cada vez más elevadas para la transmisión de energía eléctrica en estos sistemas, ha traído como consecuencia que el problema de los fenómenos transitorios originados por las descargas atmosféricas tipo impulso, que se presentan en el transformador de potencia, sean analizados no solamente a nivel de la magnitud de las sobretensiones sino también a nivel de los efectos que se producen al interior de los arrollamientos. Esto en razón, de que afectan su expectativa de vida además del posible daño a equipos que estén conectados a uno de sus arrollamientos, ya que las sobretensiones se pueden transferir al arrollamiento secundario poniendo en riesgo la continuidad del suministro de energía eléctrica [1]. Estos transitorios están dados por tipos de ondas de alta frecuencia y no periódicas - conocidas como ondas de frente rápido - que son estimadas de las sobretensiones más peligrosas. Desde este punto de vista es necesario desarrollar un modelo de los arrollamientos del transformador de potencia que considere los efectos que en ellos se producen, y permita el análisis de las tensiones transitorias con resultados confiables.

En este contexto se formula el problema general ¿Determina el modelo de los arrollamientos las tensiones transitorias transferidas en el arrollamiento secundario de un transformador de potencia frente a una tensión de impulso atmosférico?

El estudio, desde el punto de vista académico, es importante porque analiza y modela los arrollamientos incorporando la metodología de la investigación científica en base a los fundamentos de la teoría de las máquinas eléctricas, la aplicación del software para el análisis de los transitorios y los conocimientos de fabricación de transformadores de potencia. Su contribución a la innovación y desarrollo tecnológico radica en que esta metodología permitirá mejorar el diseño de los arrollamientos, dosificar adecuadamente su aislamiento e incorporar como una herramienta de análisis el software de simulación de transitorios. Centra el estudio en el modelamiento de los arrollamientos de un transformador de potencia trifásico de 15 MVA de potencia y de fabricación nacional.

Por lo expuesto se tiene como objetivo general modelar los arrollamientos para determinar las tensiones transitorias transferidas al arrollamiento secundario de un transformador de potencia frente a una tensión de impulso atmosférico; y como objetivos específicos modelar el arrollamiento primario tipo discos continuos y modelar el arrollamiento secundario tipo hélice para determinar las tensiones transitorias transferidas, en un transformador de potencia frente a una tensión de impulso atmosférico.

Para dar respuesta a la pregunta de investigación se formula la hipótesis general $\boldsymbol{H}_{\mathbf{1}}$ : El modelo de los arrollamientos determina las tensiones transitorias transferidas en un transformador de potencia frente a una tensión de impulso atmosférico. Definiéndose como variable independiente al modelo de los arrollamientos, que es la configuración del circuito eléctrico que representa a los arrollamientos en conjunto para determinar las tensiones transitorias transferidas al arrollamiento secundario de un transformador de potencia. La variable dependiente es la tensión transitoria transferida, que son los valores de la tensión que se transfiere y distribuye en toda la longitud del arrollamiento secundario del transformador de potencia.

\section{BASE TEÓRICA}

\subsection{Tensión de Impulso Atmosférico}

Los transformadores en servicio están expuestos a una variedad de esfuerzos dieléctricos. Una de las condiciones que involucran mayores solicitaciones provienen de las ondas de sobretensiones tipo rayo, que son producto de una descarga atmosférica directa o indirecta sobre el sistema eléctrico; en cualquiera de los casos, el resultado es la presencia de una onda viajera, en la línea de transmisión, que puede incidir en un borne del transformador. La forma de las ondas de sobretensión de rayo para propósitos de pruebas, han sido definidas por la International Electrotechnical Commission (IEC) [2] con el nombre de Onda de Impulso Plena, Figura 1, y Onda de Impulso Cortada en el frente. Estas ondas de frente escarpado y baja energía, cuando inciden sobre un borne del transformador, pueden ocasionar la falla del aislamiento de una de las espiras del arrollamiento afectado, lo que se manifiesta en forma de un súbito incremento de la tensión seguido por un rápido colapso del mismo [3]. 


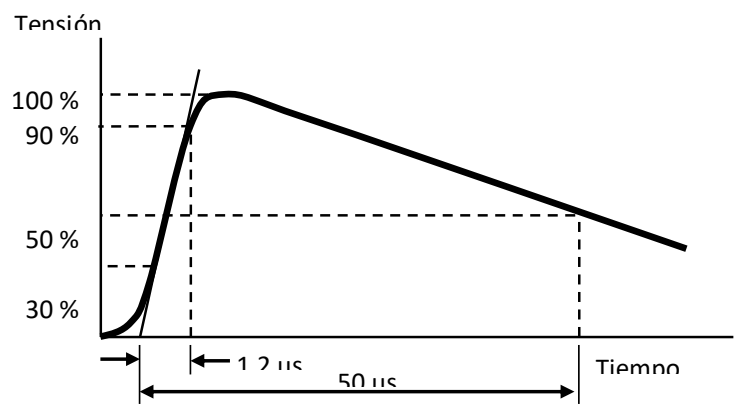

Figura 1. Onda de impulso plena [2]

\subsection{Tensiones transitorias propagadas en el arrollamiento}

La representación del circuito equivalente del arrollamiento de un transformador es muy importante para determinar su respuesta frente a la aplicación de una tensión de impulso, ya que durante los transitorios el arrollamiento está acoplado por los campos eléctrico y magnético producidos por las capacidades distribuidas y las inductancias [4]. Los transitorios pueden ser descritos, con una buena aproximación, mediante ecuaciones diferenciales simultáneas ordinarias que son resueltas por análisis numérico [5], utilizando programas computacionales, especialmente cuando las configuraciones de los arrollamientos son complejas.

En régimen transitorio la distribución inicial de tensiones se define en aproximadamente $1 \mu \mathrm{s}$, en él, la pendiente empinada del frente de onda de impulso ocasiona una distribución de tensiones no lineal en el arrollamiento afectado, que depende de la red de capacitancias parásitas presentes en el transformador. Si las capacitancias entre espiras a través del arrollamiento (capacitancias serie) fuesen uniformes, no existiendo capacitancias parásitas entre el arrollamiento y tierra (capacitancias a tierra), la tensión de impulso se distribuiría linealmente a través del arrollamiento; pero no ocurre así con cualesquiera tensión aplicada [3]. Luego, debido el efecto inductivo, continúan oscilaciones en busca de la distribución lineal dando lugar a considerables esfuerzos eléctricos, localizados en los aislamientos de ciertas espiras de los arrollamientos, hasta que la energía involucrada se disipa en la resistencia o bien ocasiona una perforación y falla. Este comportamiento explica las perforaciones observadas en el aislamiento de las primeras espiras y la gran solicitación de esfuerzo eléctrico a tierra en el primer tercio del arrollamiento del transformador de potencia.

\subsection{Tensiones Transitorias Transferidas}

El análisis de sobretensiones transitorias puede considerar la presencia de tensiones transferidas al arrollamiento secundario, ya que dichas sobretensiones en transformadores de dos arrollamientos tiene cuatro componentes [4], que son: a) el electrostático producido por la red de capacitancias, b) el electromagnético debido a la inductancia mutua entre los arrollamientos, c) las oscilaciones libres del arrollamiento secundario y d) las oscilaciones forzadas del arrollamiento secundario inducidas por las oscilaciones libres del arrollamiento primario. Estos cuatro componentes también están presentes en los terminales de los arrollamientos secundarios y terciarios cuando el primario está sujeto a la condición de impulso en un transformador de tres arrollamientos [4]. Ello implica que al modelarse los arrollamientos también debe considerarse su utilidad para interactuar con el modelo del sistema eléctrico de potencia, ya que la presencia de tensiones tipo impulso o de maniobra en el arrollamiento primario pueden ser transferidas y afectar al sistema de cargas conectadas en el arrollamiento secundario.

\section{MODELAMIENTO DE LOS ARROLLAMIENTOS}

El modelo interno del arrollamiento del transformador de potencia desarrollado en esta investigación se basa en el enfoque de la red equivalente de parámetros concentrados. Se considera como el más apropiado, para describir el comportamiento transitorio del arrollamiento, porque permite analizar y determinar la ubicación de los posibles fallos del aislamiento, en las espiras y discos, de una forma rápida y simple [6]. El modelo propuesto incluye las capacitancias de las espiras, las perdidas en corriente continua de los conductores de cobre de las espiras y las perdidas dieléctricas del material aislante. Por tanto se puede obtener suficiente precisión en el diseño del modelo de la red equivalente considerando que en los arrollamientos se agrupan los elementos: conductancia del dieléctrico, inductancia propia, inductancia mutua, resistencia de las espiras, capacitancias serie y capacitancia a tierra. Esto quiere decir que tendremos tantos elementos del circuito equivalente como discos o grupos de disco existan, considerándose a los discos, dependiendo del tipo de arrollamiento, como una espira con varias platinas en paralelo o como un conjunto de espiras. La determinación precisa del efecto de la tensión de impulso permitirá predecir las tensiones entre discos o secciones, y utilizar estos valores para diseñar el aislamiento interno del arrollamiento.

En el caso de los transformadores, que operan en baja frecuencia, como por ejemplo $60 \mathrm{~Hz}$, la corriente de 
desplazamiento a través de la conductancia - que representa las pérdidas de los dieléctricos del arrollamiento - puede aproximarse a cero, y los arrollamientos se pueden modelar solo con resistencias, inductancias propias e inductancias mutuas [6]. Pero a altas frecuencias, como son las sobretensiones por descargas atmosféricas tipo impulso, el efecto de las corrientes de desplazamiento desde una sección de arrollamiento a otra deben ser tomadas en consideración y por tanto, las capacitancias y las conductancias entre arrollamientos primario y secundario tienen que ser incluidas en el modelo del arrollamiento del transformador [6]. Para altas frecuencias, cada arrollamiento del transformador de potencia se puede considerar como una línea de transmisión, en donde los parámetros concentrados equivalentes deben incorporar las características eléctricas y magnéticas de las espiras. Sin embargo, el modelo espira-espira tiene alta complejidad y requiere excesivo esfuerzo computacional, lo que se puede evitar agrupando las espiras en bloques o secciones [7]. Considerando lo expuesto se propone el modelo integral de los arrollamientos que se describe a continuación, para determinar las tensiones transferidas de un arrollamiento al otro.

El arrollamiento se divide en bloques, los cuales tendrán una conformación que dependerá del tipo de arrollamiento. Así se tiene que si es un arrollamiento tipo hélice (característico de baja tensión), el bloque estará formado por espiras; mientras que si es del tipo discos continuos o discos tipo espiras intercaladas (característicos de alta tensión), el bloque estará formado por discos. Para una mayor precisión en los resultados los arrollamientos deben contener la mayor cantidad posible de bloques. Cada bloque se representa por un circuito equivalente de parámetros concentrados, en el cual se incorporan el acoplamiento magnético y capacitivo entre bloques correspondientes al arrollamiento de alta y baja tensión [6]. El circuito eléctrico equivalente de un par de bloques se detalla en la Figura 2, en el que los subíndices " $p$ " $y$ " $s$ " indican que los parámetros concentrados pertenecen al bloque del arrollamiento primario y al bloque del arrollamiento secundario respectivamente. Así se tiene que para cada bloque del arrollamiento, $R p_{k}$ y $R s_{l}$ son las resistencias debido a las pérdidas en el arrollamiento, $L p_{k}$ y $L s_{l}$ son las inductancias propias, $C p_{k}$ y $C s_{l}$ son las capacitancias serie, $\operatorname{Cop}_{k}$ y $\operatorname{Cos}_{l}$ son las capacitancias a tierra, $G_{o p}$ y $\mathrm{Gos}_{l}$ son las conductancias a tierra debido a las perdidas dieléctricas, $M p s_{k l}$ es la inductancia mutua, $\operatorname{Cops}_{k l}$ es la capacitancia entre arrollamientos y $G_{0 p s} s_{k l}$ es la conductancia entre arrollamientos debido a las perdidas dieléctricas.

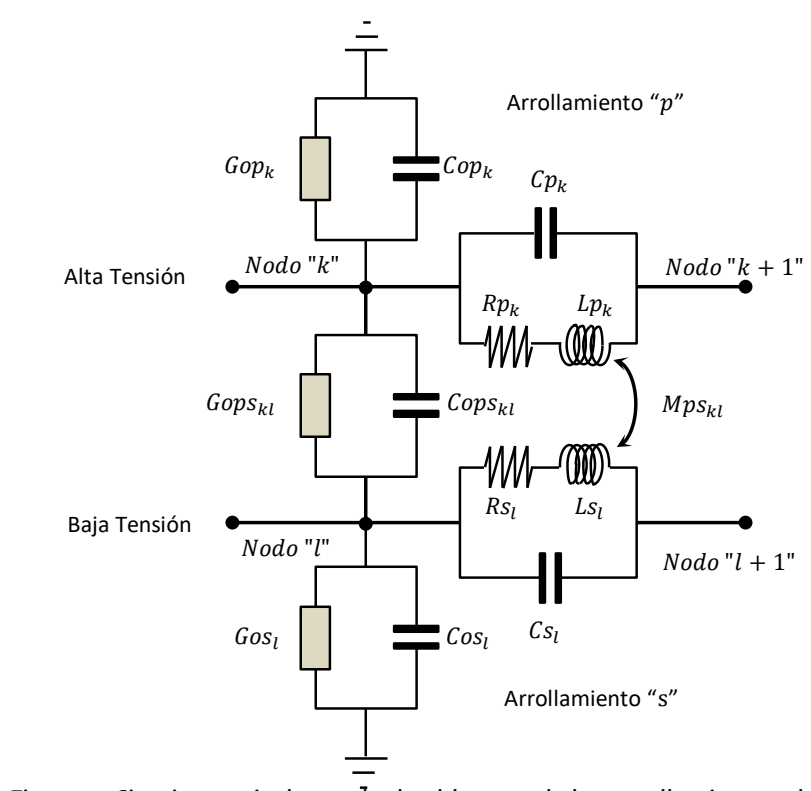

Figura 2. Circuito equivalente de dos bloques de los arrollamientos de alta tensión y baja tensión [6]. $k$ y $l$ son los números de nodos.

\section{METODOLOGÍA}

La unidad de análisis de la investigación estuvo constituida por las tensiones transitorias que se presentan en las espiras de los arrollamientos del transformador de potencia frente a la aplicación de una tensión tipo impulso atmosférico. La muestra estuvo constituida por un transformador de potencia de 15 MVA y relación de transformación de 56/10 kV con conmutador bajo carga y de fabricación nacional. Las características constructivas de los arrollamientos se describen sucintamente a continuación.

El arrollamiento primario de alta tensión se compone del arrollamiento principal AT y del arrollamiento de regulación fina REG. El arrollamiento de alta tensión es a discos continuos y consta de 62 discos con 13 espiras por disco. El arrollamiento de regulación es del tipo hélice a espiras intercaladas. El arrollamiento secundario de baja tensión se compone del arrollamiento principal $B T$, que es del tipo hélice y consta de 83 espiras, que vienen a ser los discos del mismo. Cada arrollamiento tiene como terminales de entrada $1 \mathrm{P}$ de $A T, 1 \mathrm{~S}$ de $B T, K$ de REG y como terminales de salida $\mathrm{P}$ de $A T$, $S$ de $B T$ y $F$ de REG. Dado que las espiras se agrupan en varios bloques, como se modeló en la Fig. 2, se consideraran 62 bloques tanto para el arrollamiento de AT como para el arrollamiento de BT, ver Tabla 1, con lo que se obtiene un modelo que se considera preciso para el análisis transitorio. En el arrollamiento de AT cada uno de los 62 bloques estará constituido por un disco mientras que en el arrollamiento de BT se ha considerado que los primeros 41 bloques estarán conformados por un solo disco y los 21 bloques restantes por dos discos. El arrollamiento de regulación fina REG no será considerado en el análisis ya 
que las simulaciones se harán para las tensiones nominales del transformador. En base al modelo propuesto se llevaron a cabo las siguientes fases operativas:

a) Se aplicó un método auto desarrollado con formulaciones convencionales para calcular los parámetros eléctricos de la red equivalente de los arrollamientos, como fueron: Inductancias de los arrollamientos [8][1], Capacitancia Serie [4], Capacitancia a Tierra [4][9];

b) Se utilizaron los resultados de las pruebas Resistencia de los Arrollamientos, Tabla 2, y Factor de Potencia del Aislamiento, Tabla 3, para determinar los valores de las resistencias de las espiras y de las conductancias del dieléctrico;

c) Utilizando el modelo integral de los arrollamientos se simuló la aplicación de una tensión de impulso atmosférico analizándose sus efectos, en las tensiones transitorias propagadas en cada arrollamiento y en las tensiones transitorias transferidas al arrollamiento secundario, con el uso del software ATP Electromagnetic Transients Program, ATP Draw [10].

Tabla 1. Conformación de bloques de los arrollamientos

\begin{tabular}{|c|c|c|c|c|}
\hline Arrollamiento & $\begin{array}{c}\text { Número } \\
\text { de } \\
\text { Bloques }\end{array}$ & $\begin{array}{c}\text { Número } \\
\text { de Discos } \\
\text { por } \\
\text { Bloque }\end{array}$ & $\begin{array}{c}\text { Número } \\
\text { de } \\
\text { Espiras } \\
\text { por } \\
\text { Bloque }\end{array}$ & $\begin{array}{c}\text { Tensión } \\
\text { de } \\
\text { Impulso }\end{array}$ \\
\hline Alta Tensión & 41 & 1 & 13 & \begin{tabular}{c}
$325 \mathrm{kV}$ \\
\cline { 2 - 4 } Baja Tensión
\end{tabular} \\
\cline { 2 - 4 } & 21 & 1 & 13 & $1,2 / 50 \mu \mathrm{s}$ \\
\hline
\end{tabular}

Tabla 2. Resistencia de los arrollamientos

\begin{tabular}{|l|c|c|c|c|c|}
\hline Medida & $\begin{array}{c}\text { Temperatura } \\
\text { Referida } \\
\left({ }^{\circ} \mathrm{C}\right)\end{array}$ & \multicolumn{4}{|c|}{$\begin{array}{c}\text { Resistencia del Arrollamiento } \\
\text { (miliohmios) }\end{array}$} \\
\hline \multirow{2}{*}{$\begin{array}{l}\text { Alta } \\
\text { Tensión }\end{array}$} & 75 & $1 \mathrm{U}-1 \mathrm{~V}$ & $1 \mathrm{~V}-1 \mathrm{~W}$ & $1 \mathrm{~W}-1 \mathrm{~V}$ & \multirow{2}{*}{1069,70} \\
\cline { 3 - 5 } & 1064,30 & 1050,00 & 1065,00 & \\
\hline $\begin{array}{l}\text { Baja } \\
\text { Tensión }\end{array}$ & 75 & $2 \mathrm{U}-2 \mathrm{~N}$ & $2 \mathrm{~V}-2 \mathrm{~N}$ & $2 \mathrm{~W}-2 \mathrm{~N}$ & \multirow{2}{*}{10,13} \\
\cline { 3 - 5 } & & 10,10 & 10,00 & 10,30 & \\
\hline
\end{tabular}

Tabla 3. Factor de potencia del aislamiento

\begin{tabular}{|l|c|c|c|c|}
\hline \multicolumn{1}{|c|}{ Medida } & $\begin{array}{c}\text { Tensión } \\
\text { de } \\
\text { prueba } \\
(\mathrm{kV})\end{array}$ & $\begin{array}{c}\text { Corriente } \\
(\mathrm{mA})\end{array}$ & $\begin{array}{c}\text { Potencia } \\
\text { (Watts) }\end{array}$ & $\begin{array}{c}\text { Factor de } \\
\text { potencia } \\
\text { corregido } \\
(\%)\end{array}$ \\
\hline $\begin{array}{l}\text { Entre alta } \\
\text { tensión y tierra }\end{array}$ & 10,0050 & 9,1210 & 0,2490 & $0,26^{*}$ \\
\hline $\begin{array}{l}\text { Entre alta y baja } \\
\text { tensión }\end{array}$ & 10,0040 & 14,1130 & 0,2500 & $0,17^{*}$ \\
\hline $\begin{array}{l}\text { Entre baja } \\
\text { tensión y tierra }\end{array}$ & 6,0020 & 22,7510 & 0,5970 & $0,25^{*}$ \\
\hline
\end{tabular}

$\left(^{*}\right)$ : [11]; kV: Kilovoltio; mA: miliamperio.

La configuración de las conexiones de una fase para la simulación se muestra en la Figura 3; en donde el impulso de tensión se aplica al terminal $1 \mathrm{P}$ de $A T$, estando el terminal $\mathrm{P}$ de $A T$ conectado al terminal positivo $O$ de $R E G$; con los terminales $F$ de REG y $S$ de $B T$ conectados a tierra. El terminal $1 \mathrm{~S}$ de BT está desconectado. 

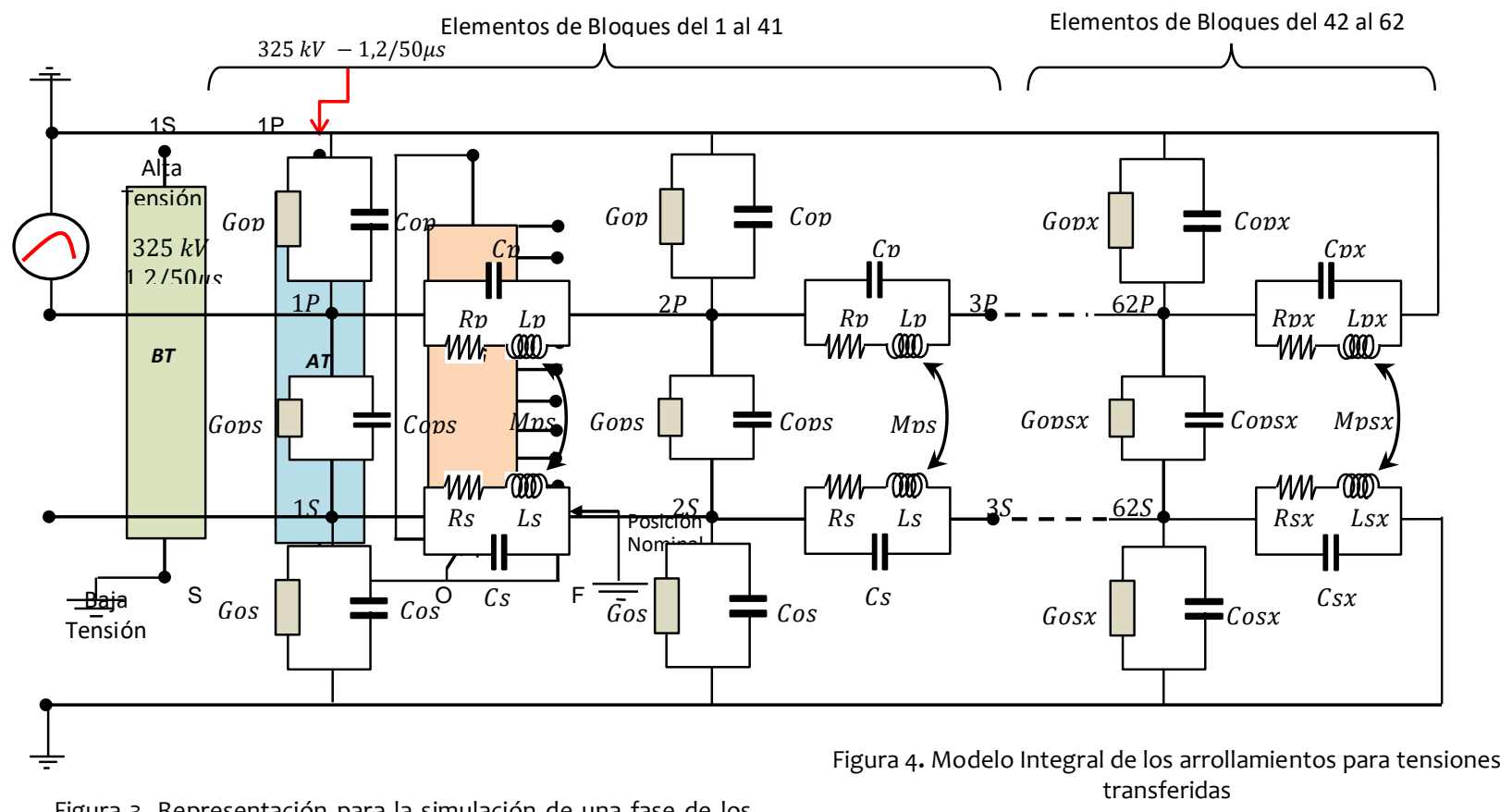

Figura 3. Representación para la simulación de una fase de los transferidas arrollamientos del transformador de potencia.

\section{RESULTADOS}

Para determinar la distribución de tensiones transitorias transferidas, se utilizó el circuito equivalente del modelo integral de los arrollamientos de una de las fases del transformador de potencia que se muestra en la Figura 4. Los valores calculados de los elementos que conforman el circuito equivalente del modelo integral de los arrollamientos se presentan en la Tabla 4.

Tabla 4. Elementos del circuito equivalente del modelo integral de los arrollamientos

\begin{tabular}{|c|c|c|c|c|}
\hline \multirow{3}{*}{ Denominación } & \multicolumn{4}{|c|}{ Bloques del Modelo de los Arrollamientos } \\
\hline & \multicolumn{2}{|c|}{1 al 41} & \multicolumn{2}{|c|}{42 al 62} \\
\hline & Notación & Valor & Notación & Valor \\
\hline \multicolumn{5}{|l|}{ Arrollamiento de Alta Tensión } \\
\hline Resistencia del arrollamiento & $R p$ & $17,2530 \mathrm{~m} \Omega$ & $R p x$ & $17,2530 \mathrm{~m} \Omega$ \\
\hline Inductancia del arrollamiento & $L p$ & $1,4580 \mathrm{mH}$ & $\operatorname{Lpx}$ & $1,4580 \mathrm{mH}$ \\
\hline Capacitancia a tierra entre arrollamiento y tanque & Cop & $11,8600 p F$ & Copx & $11,8600 p F$ \\
\hline Capacitancia serie del arrollamiento & $C p$ & $1424,7600 p F$ & $\operatorname{Cpx}$ & $1424,7600 p F$ \\
\hline Conductancia a tierra entre arrollamiento y tanque & Gop & $13,1800 p S$ & Gopx & $13,1800 p S$ \\
\hline \multicolumn{5}{|l|}{ Entre Arrollamientos de Alta Tensión y Baja Tensión } \\
\hline Inductancia mutua entre arrollamientos & Mps & $0,0289 m H$ & Mpsx & $0,1178 m H$ \\
\hline Capacitancia a tierra entre arrollamientos & Cops & $17,3500 p F$ & Cps $x$ & $24,7600 p F$ \\
\hline Conductancia a tierra entre arrollamientos & Gops & $11,6400 p S$ & Gpsx & $16,6600 p S$ \\
\hline
\end{tabular}




\begin{tabular}{|c|c|c|c|c|}
\hline Arrollamiento de Baja Tensión & & & & \\
\hline Resistencia del arrollamiento & $R s$ & $0,1220 m \Omega$ & $\operatorname{Rs} x$ & $0,2440 \mathrm{~m} \Omega$ \\
\hline Inductancia del arrollamiento & $L s$ & $0,0080 \mathrm{mH}$ & $L s \mathrm{x}$ & $0,0160 m H$ \\
\hline $\begin{array}{l}\text { Capacitancia a tierra entre arrollamiento y núcleo } \\
\text { magnético }\end{array}$ & $\operatorname{Cos}$ & $22,3900 p F$ & $\operatorname{Cos} x$ & $44,7800 \mathrm{pF}$ \\
\hline Capacitancia serie del arrollamiento & Cs & $740,3600 p F$ & $\operatorname{Cs} x$ & $370,1800 p F$ \\
\hline $\begin{array}{l}\text { Conductancia a tierra entre arrollamiento y núcleo } \\
\text { magnético }\end{array}$ & Gos & $21,7700 p S$ & Gosx & $43,5200 p S$ \\
\hline
\end{tabular}

Las unidades expresadas en: miliohmios $(m \Omega)$; milihenrios $(m H)$; picofaradios $(p F)$; picosiemens $(p S)$.

En la simulación de transitorios con el software ATP Electromagnetic Transients Program, ATP Draw [10] se aplicó al terminal de entrada del arrollamiento de alta tensión una tensión de onda plena de $325 \mathrm{kV}$ a 1,2/50 $\mu \mathrm{s}$ [12] con el arrollamiento de baja tensión a circuito abierto y uno de sus bornes puesto a tierra. El tiempo de evaluación fue de $50 \mu$ s obteniéndose los resultados que se presentan a continuación.

\subsection{Tensiones Transitorias en el Arrollamiento de Alta Tensión}

Las tensiones transitorias en diferentes puntos de evaluación del arrollamiento de alta tensión se muestran en la Figura 5, Figura 6, Figura 7, Figura 8, Figura 9 y Figura 10.

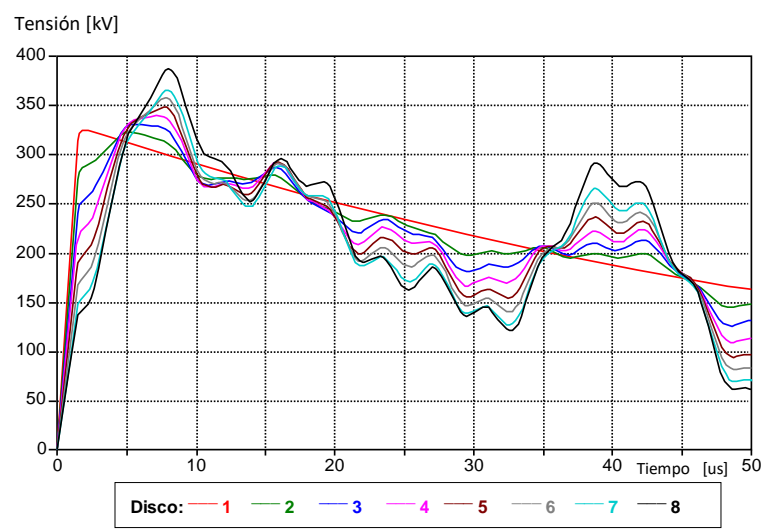

Figura 5. Tensión transitoria a tierra de discos de AT. 


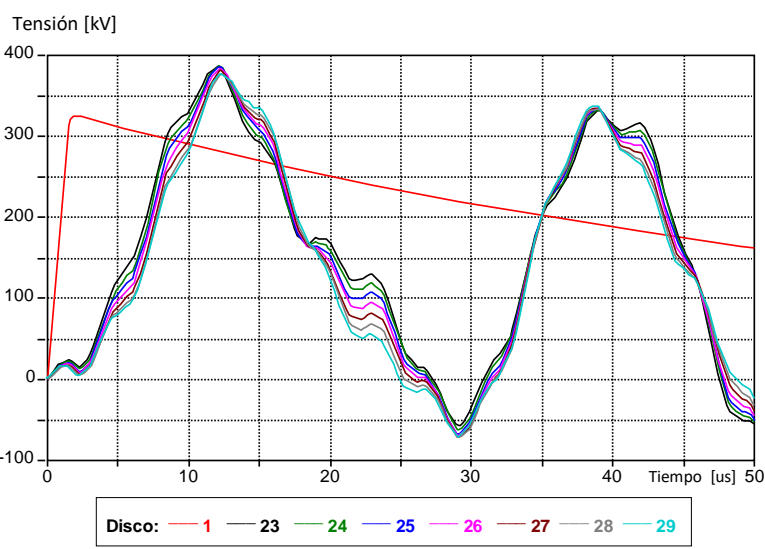

Figura 6. Tensión transitoria a tierra de discos de AT.

Tensión [kV]

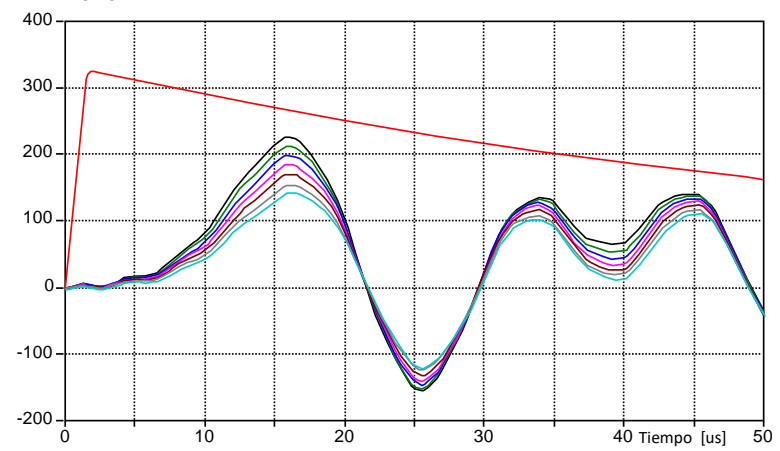

Disco: $-1-49-50-51-52-53-54-55$

Figura 7. Tensión transitoria a tierra de discos de AT. Tensión [kV]

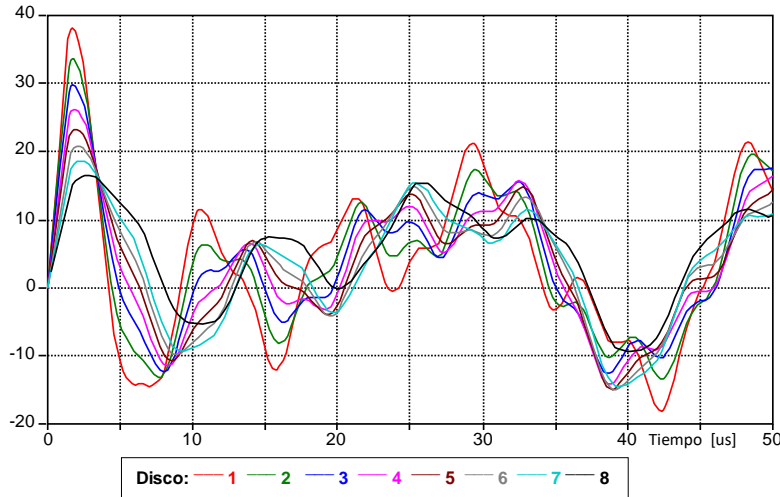

Figura 8. Tensiones transitorias distribuidas en discos de AT. Tensión [kV]

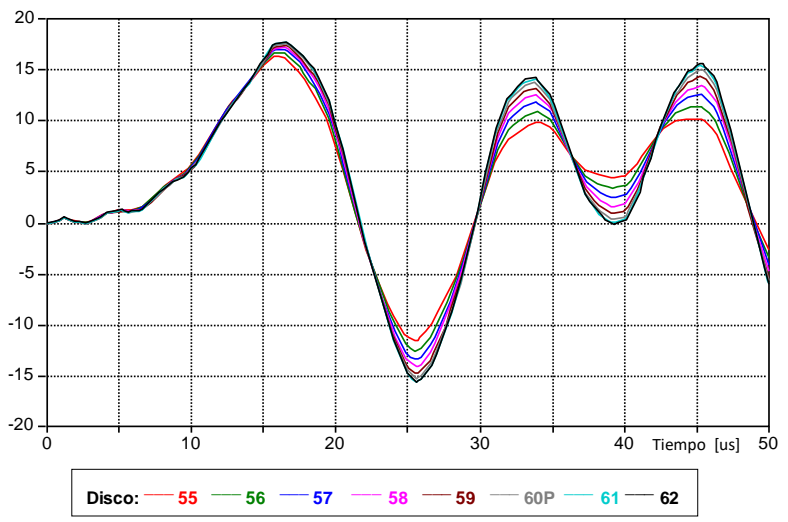

Figura 9. Tensiones transitorias distribuidas en discos de AT.

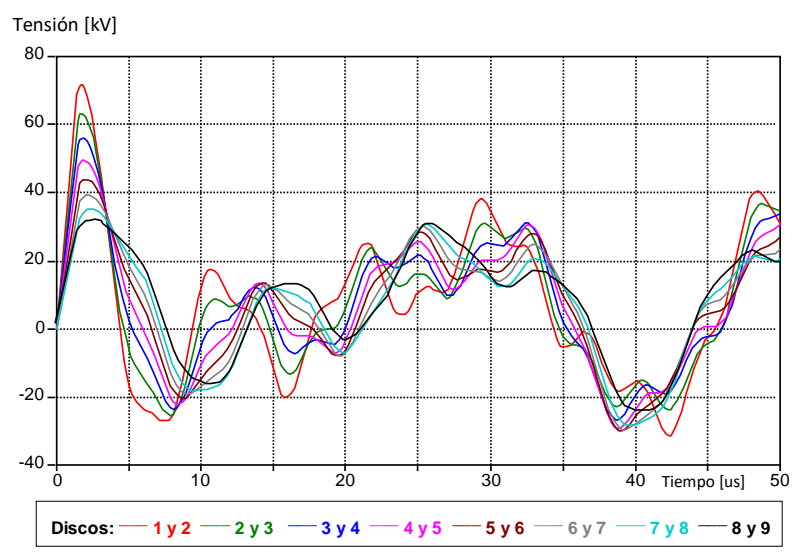

Figura 10. Tensiones transitorias entre discos contiguos de AT.

\subsection{Tensiones Transitorias Transferidas al Arrollamiento de Baja Tensión}

Las tensiones transitorias en diferentes puntos de evaluación del arrollamiento de baja tensión se muestran en la Figura 11, Figura 12, Figura 13, Figura 14 y Figura 15.

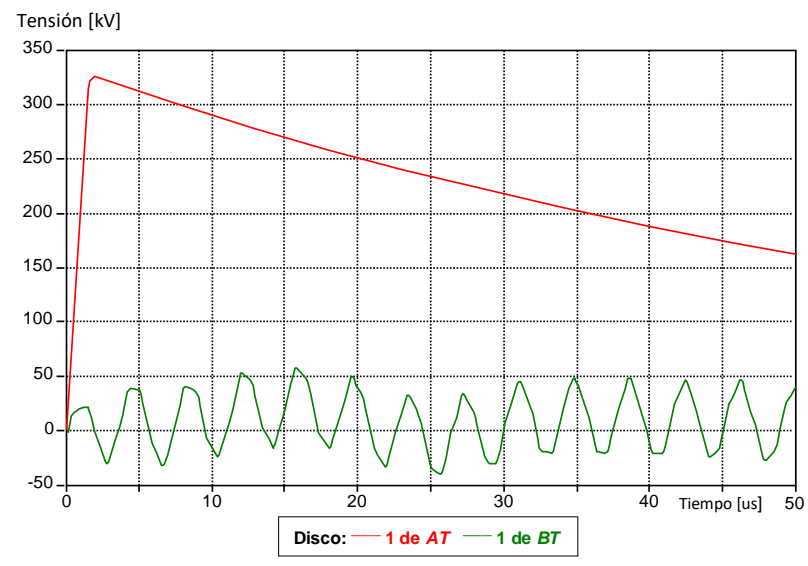

Figura 11. Tensión transitoria a tierra de discos de AT y BT.

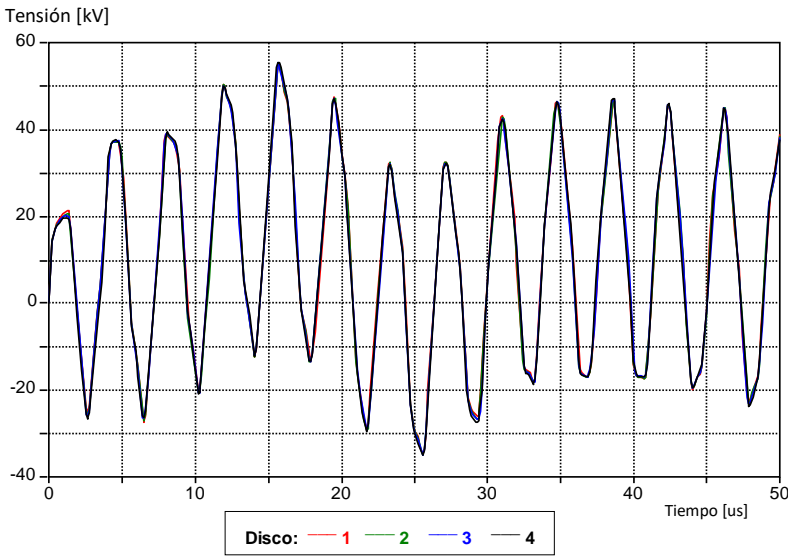

Figura 12. Tensión transitoria a tierra de discos de BT. 


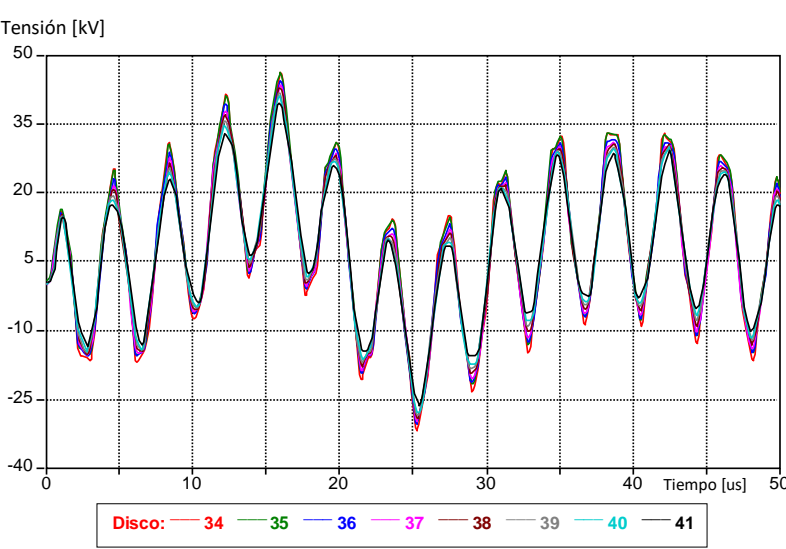

Figura 13. Tensión transitoria a tierra de discos de BT.

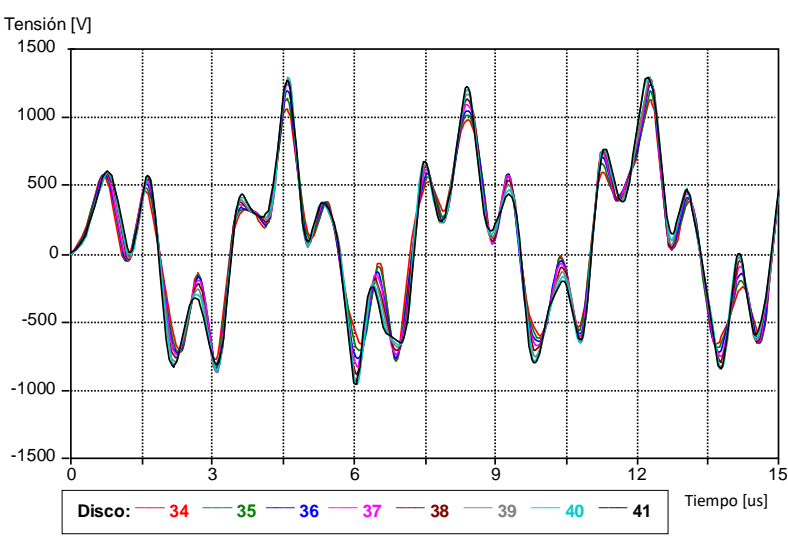

Figura 14. Tensión transitoria distribuida en discos de BT.

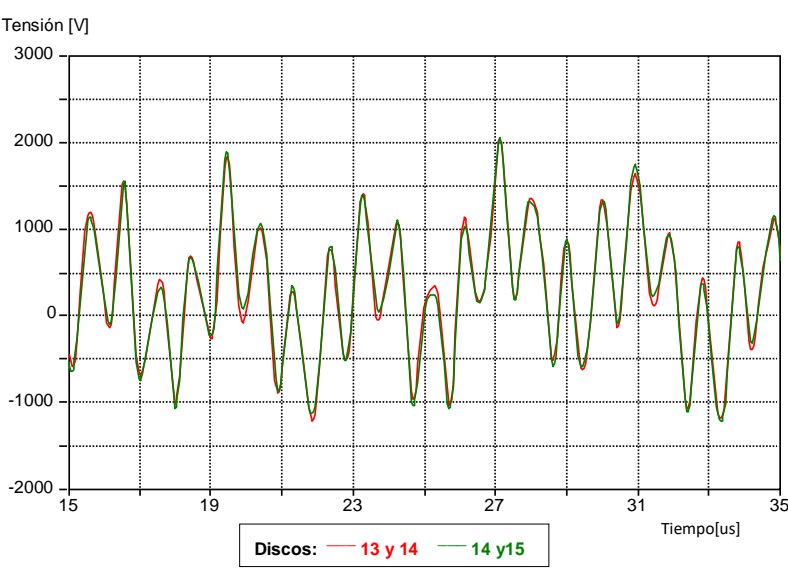

Figura 15. Tensión transitoria entre discos contiguos de BT.

Por ser las tensiones transitorias del arrollamiento de alta tensión significativamente superiores a las del arrollamiento de baja tensión, se presentan para el análisis, en la Tabla 5, las tensiones transitorias máximas entre discos y entre espiras del arrollamiento de alta tensión
Tabla 5. Tensiones transitorias máximas en arrollamiento de alta tensión

\begin{tabular}{|c|c|c|c|c|}
\hline \multirow{2}{*}{$\begin{array}{l}\text { Impulso de } \\
\text { Onda Plena }\end{array}$} & \multirow{2}{*}{$\begin{array}{l}\text { Punto de } \\
\text { Evaluación }\end{array}$} & \multicolumn{3}{|c|}{ Esfuerzo Dieléctrico Máximo } \\
\hline & & $\begin{array}{c}\text { Tensión } \\
(\mathrm{kV})\end{array}$ & $\begin{array}{c}\text { Tiempo } \\
(\mu \mathrm{s})\end{array}$ & Discos \\
\hline \multirow{2}{*}{$\begin{array}{c}325 \mathrm{kV} \\
1,2 / 50 \mu \mathrm{s}\end{array}$} & $\begin{array}{c}\text { Entre Discos } \\
\text { Contiguos }\end{array}$ & 71,30 & 1,70 & 1 y 2 \\
\hline & $\begin{array}{c}\text { Entre } \\
\text { Espiras }\end{array}$ & 2,95 & 1,60 & 1 y 2 \\
\hline
\end{tabular}

Fuente: Resultados de Simulaciones [10].

\section{ANÁLISIS E INTERPRETACIÓN}

Los resultados de las simulaciones utilizando el modelo integrado de los arrollamientos, muestran en general un proceso de intercambio de energía entre los elementos inductivos y capacitivos con amortiguación moderada por el efecto de las resistencias y conductancias de cada arrollamiento, que se explican a continuación.

En el arrollamiento de alta tensión se aprecia gráficamente, Figura 5 y Figura 6, que los valores de las tensiones a tierra tienen oscilaciones con grandes amplitudes - que se extienden a lo largo de los 30 primeros discos del arrollamiento - producidos por las capacitancias e inductancias propias del arrollamiento y por la inductancia mutua entre arrollamientos. Se evidencia también un fuerte efecto amortiguador proveniente de las resistencias de las espiras y de las conductancias a tierra del arrollamiento, que se nota claramente por la disminución de la amplitud de las oscilaciones y frecuencias de las tensiones transitorias a tierra a partir del disco 49, Figura 7, como consecuencia de la disminución de la longitud del arrollamiento afectado. De igual forma este efecto se manifiesta para las tensiones distribuidas en los discos del arrollamiento, como se observa en la Figura 8 y Figura 9.

En la gráfica de la Figura 6, los discos 23, 24 y 25 del arrollamiento de alta tensión, que contienen a las espiras 286 a 325, soportan la máxima tensión a tierra, alcanzando un valor de $386,90 \mathrm{kV}$ en el tiempo de 12,14 $\mu$ s. También se puede apreciar, en la Figura 8, que el primer disco de este arrollamiento soporta la máxima tensión distribuida, alcanzando un valor de 38,24 kV en el tiempo de 1,65 $\mu \mathrm{s}$. En la Figura 10, se observa que entre los discos 1 y 2 del arrollamiento de alta tensión, que contienen a las espiras de la 1 a la 26, se presenta la máxima tensión entre discos contiguos, alcanzando un valor de 71,30 kV en el tiempo de 1,70 $\mu$ s; tensión que debe soportar el aceite dieléctrico que ocupa este espacio entre discos y el espesor del papel aislante de las espiras que se encuentran en esta zona. Así mismo, 
de la Tabla 5 se obtiene que la tensión máxima entre espiras es de 2,95, tensión que debe ser soportada por el espesor del papel aislante que las separa. Se aprecia en la Figura 11, que cuando la tensión de impulso alcanza el valor de $261 \mathrm{kV}$ en los terminales de entrada del arrollamiento de alta tensión, se produce una tensión oscilatoria de 20,47 kV en los terminales del arrollamiento de baja tensión; lo que corrobora, con aproximación, el valor de la relación del número de espiras entre arrollamientos.

En el arrollamiento de baja tensión para los 34 primeros discos, se obtienen valores - tanto de la tensión a tierra de los discos, Figura 12 y Figura 13, como para las tensiones distribuidas en los discos, Figura 14 que revelan un considerable comportamiento oscilatorio producido por el efecto de las capacitancias, inductancias propias e inductancias mutuas de los arrollamientos, ya que el valor de la resistencia de las espiras es imperceptible. Esto se hace muy notorio a lo largo de todo el arrollamiento, evidenciándose también un moderado amortiguamiento a partir del disco 34, conforme la tensión se propaga, ya que las amplitudes de las oscilaciones disminuyen, sin variar su frecuencia de oscilación, debido al intercambio de energía y por el efecto disipador de la conductancia mutua y resistencia del arrollamiento. En este arrollamiento, Figura 12, se observa que los discos 1, 2, 3 y 4 soportan la máxima tensión a tierra, alcanzando un valor aproximado de 55,17 kV en el tiempo de $15,70 \mu$ s. Así mismo se puede apreciar, en la Figura 14, que los discos 38, 39, 40 y 41 del arrollamiento de baja tensión soportan la máxima tensión distribuida en los discos, alcanzando el valor de 1,30 kV en el tiempo de 12,22 $\mu \mathrm{s}$.

En la Figura 15, se observa que entre los discos 13 y 14; 14 y 15 del arrollamiento de baja tensión se presenta la máxima tensión transitoria entre discos contiguos, alcanzando un valor de 2,04 kV en el tiempo de 27,1 $\mu$ s. Tensión que debe soportar el aceite dieléctrico que ocupa este espacio entre discos y el espesor del papel aislante de las espiras que se encuentran en esta zona.

Por lo expuesto, el análisis de las sobretensiones transitorios se puede enfocar considerando que cada arrollamiento es un circuito sub amortiguado RLC, en los cuales la amplitud de la tensión y el tiempo del período de las oscilaciones definen a la señal de respuesta. Así se tiene que en el arrollamiento de alta tensión, si bien la sobretensión transitoria lógicamente tiene mayor amplitud, el tiempo del período de oscilación es mayor que en el arrollamiento de baja tensión, debido a que su valor es, aproximadamente, inversamente proporcional a la raíz cuadrada del producto de los valores de la inductancia y capacitancia de cada arrollamiento.
A partir de los valores de la Tabla 5, se puede determinar el esfuerzo dieléctrico máximo al que es sometido el sistema de aislamiento del arrollamiento de alta tensión. Por ejemplo, el caso del aislamiento entre discos contiguos que tienen que soportan una tensión máxima de 71,30 kV. De acuerdo al diseño del arrollamiento del transformador, la separación con aceite entre discos es de 4,5 mm, y según el procedimiento de cálculo [6] con $K=2$, que es una constante que depende de la frecuencia de la tensión aplicada, la rigidez dieléctrica admisible del espacio con aceite entre los discos se evalúa como:

$$
\begin{gathered}
E_{\text {adm.aceite }}=18 * k * d_{a c}^{-0,37} \\
E_{\text {adm.aceite }}=18 * 2 * 4,5^{-0,37}=20,63 \frac{\mathrm{kV}}{\mathrm{mm}}
\end{gathered}
$$

Considerando que entre discos también existe papel de celulosa que cubre a las platinas de cobre, se tendrá que calcular la rigidez dieléctrica máxima que soportan el aceite y el papel de celulosa. Así se tiene que la rigidez dieléctrica en la parte del aislamiento entre discos contiguos se puede calcular considerándolo, con buena aproximación, como homogéneo; y, según [13], al aplicar una tensión $\boldsymbol{U}$ a todo el ancho del aislamiento constituido por dos tipos de material aislante la rigidez dieléctrica para el aceite $E_{\text {aceite }}$ y para el papel de celulosa $E_{\text {papel }}$ en $\mathrm{kV} / \mathrm{mm}$, será:

$$
\begin{gathered}
E_{\text {aceite }}=\frac{U}{\varepsilon_{a c}\left[\frac{d_{a c}}{\varepsilon_{a c}}+\frac{d_{p}}{\varepsilon_{p}}\right]} \\
E_{\text {papel }}=E_{\text {aceite }}\left[\frac{\varepsilon_{a c}}{\varepsilon_{p}}\right]
\end{gathered}
$$

Para el análisis se considera que $U=71,30 \mathrm{kV}$, $d_{a c}=4,5 \mathrm{~mm}$ es el espesor del aceite, $d_{p}=1,1 \mathrm{~mm}$ es el biespesor del papel de celulosa, $\varepsilon_{a c}=2,20$ es la permitividad del aceite y $\varepsilon_{p}=3,05$ es la permitividad del papel de celulosa. Si la rigidez dieléctrica admisible es $E_{\text {adm.aceite }}=20,63 \mathrm{kV} / \mathrm{mm}$ y $E_{\text {adm.papel }}=40 \mathrm{kV} /$ $\mathrm{mm}$; de las ecuaciones (1) y (2) se obtiene:

$$
\begin{gathered}
E_{\text {aceite }}=13,47 \frac{\mathrm{kV}}{\mathrm{mm}}<E_{\text {adm. aceite }} \\
E_{\text {papel }}=9,71 \frac{\mathrm{kV}}{\mathrm{mm}}<E_{\text {adm.papel }}
\end{gathered}
$$

Por tanto, al ser la rigidez dieléctrica máxima del aceite y del papel de celulosa menores que el valor de la rigidez dieléctrica admisible del aceite y del papel se concluye, que el material aislante del espacio de separación entre discos contiguos soporta las sobretensiones transitorias originadas por el impulso de onda plena. 
Con respecto al problema general planteado en la investigación y considerando los resultados obtenidos en las simulaciones, se puede confirmar la validez del modelo integral de los arrollamientos ya que se logran determinar las tensiones propagadas y transferidas, y comprobar su utilidad en el cálculo de los esfuerzos dieléctricos máximos que soportan los aislamientos de los arrollamientos. Por tanto se comprueba y acepta la hipótesis general.

Para casos prácticos de diseño del aislamiento interno se debe considerar que la forma de onda estándar de 1,2/50 $\mu$ s, del impulso atmosférico, se distorsiona al ingresar al arrollamiento del transformador [14] produciéndose variaciones tanto en el tiempo del frente como en el tiempo de cola. Si estos se incrementaran se producirá una disminución con tendencia exponencial de la tensión de ruptura del aislamiento sólido [14]. Este comportamiento se puede analizar con el modelo de integral de los arrollamientos propuesto, ya que permite la simulación con ondas de impulso de diferente forma.

\section{CONCLUSIONES}

El principal aporte del estudio ha sido comprobar la utilidad del modelo integral de los arrollamientos de un transformador de potencia, para analizar y determinar tanto las tensiones transitorias propagadas en el arrollamiento primario como las tensiones transitorias transferidas al arrollamiento secundario, frente a una tensión de impulso atmosférico. Según ello las conclusiones que se derivaron del estudio son las siguientes:

Se probó la hipótesis general $H_{1}$, por tanto se infiere que conforme el modelamiento de los arrollamientos sea más detallado en su configuración como una red eléctrica de parámetros concentrados, más precisa será la determinación de las tensiones transitorias propagadas y transferidas.

El modelo propuesto y la metodología desarrollada ofrecen una herramienta práctica y adecuada para el análisis en el dominio del tiempo de las sobretensiones transitorias y para estimar y evaluar los esfuerzos dieléctricos a los que son sometidos los aislamientos; pudiendo aplicarse en la etapa de diseño del transformador de potencia.
Se podría hacer extensiva la utilidad del modelo propuesto asociándolo con el modelo de una línea de transmisión para simular diferentes tipos de fallos y estimar las tensiones transitorias propagadas $y$ transferidas a los arrollamientos del transformador de potencia.

\section{REFERENCIAS}

[1] Escamilla J 2008 Modelado para Análisis de la Transferencia de Sobretensiones Inducidas por Descargas Atmosféricas en Sistemas de Distribución. [tesis doctoral]. México: Instituto Politécnico Nacional p 118

[2] IEC International Standard 60-1 1989-11. High-voltage test techniques - Part 1: General definitions and test requirements

[3] Nutt W J Mc. April 1976. Application of distribution and power transformers. IEEE Tutorial Course

[4] Kulkarni S V and Khaparde S A 2004 Transformer Engineering. Marcel Dekker Inc.: New York. pp 282-307

[5] Smajic J, Steinmetz T, Rüegg M, Tanasic Z, Obrist R, Tepper J, Weber B and Carlen M. Feb. 2014. Simulation and Measurement of Lightning-Impulse Voltage Distributions Over Transformer Windings. IEEE Transactions on Magnetics. 50(2): pp 553 - 556 https://www.researchgate.net/publication/3478276

[6] Álvarez C 2014 Estudio de la respuesta en muy alta frecuencia en transformadores de potencia [tesis doctoral] Vigo: Universidad de Vigo p 204

[7] Shibuya Y, Matsumoto T and Teranishi T. June 19-23. 2005. Modelling and Analysis of Transformer Winding at High Frequencies. Proc. International Conference on Power Systems Transients (IPST'05). Montreal. Canada

[8] Corrales M J. 1975. Cálculo Industrial de Máquinas Eléctricas. Universidad Politécnica de Barcelona. Tomo I: pp. 218-220 pp. 276278

[9] Del Vecchio R, Paulin B, Feghali P, Shah D and Ahuja R 2002 Transformer Design Principles. CRC Press

[10] The graphical preprocessor to ATP Electromagnetic Transients Program. ATP Draw. http://www.atpdraw.net/

[11] IEEE Std 1999. IEEE Standard Test Code for Liquid-Inmersed Distribution, Power, and Regulating Transformers - Revision of IEEE Std

[12] IEC International Standard 2013 Power Transformers - Part 3: Insulation levels, dielectric tests and external clearances in air.

[13] Karsai K, Kerényi D and Kiss L 1987 Large Power Transformers. Elsevier Science Publishing Company Inc. pp 250-255

[14] Ni H, Li Y, Yuan W, Gao M, Ding Y, Zhao Y, Zhang Q and Wang Z 2017 Dependence of the Impulse Strength of Typical Insulation Structures in Oil-immersed Transformer on Waveshapes. $19^{\text {th }}$ IEEE International Conference on Dielectric Liquids (ICDL). Manchester. United Kingdom pp 25-29

Los artículos publicados por TECNIA pueden ser compartidos a través de la licencia Creative Commons: CC BY-NC-ND 2.5 Perú. Permisos lejos de este alcance pueden ser consultados a través del correo revistas@uni.edu.pe 